\title{
Topology Optimization of Compliant Mechanisms Using Guide-Weight Method
}

\author{
Liping Wang ${ }^{\mathrm{a}}$, Huayang $\mathrm{Xu}^{\mathrm{b}}$ and Liwen Guan ${ }^{\mathrm{c}}$ \\ Department of Mechanical Engineering, Tsinghua University, Beijing 100084, China \\ alpwang@tsinghua.edu.cn, bxuhy14@mails.tsinghua.edu.cn, cguanlw@mail.tsinghua.edu.cn
}

Keywords: Topology optimization, Guide-Weight method, Compliant mechanisms.

\begin{abstract}
This paper introduces the Guide-Weight method into solving the topology optimization problems of compliant mechanisms. Guide-Weight method is a kind of Optimality Criteria methods, and it possesses the characteristics of clear physical meaning, high computational efficiency and great generality, which is definitely suitable for solving this class of problems. First, all theoretical derivations of GW are carried out. Furthermore, the relative spring stiffness ratio is proposed to update the input and output port element's stiffness value, and this approach exhibits high efficiency and strong robustness. Lastly, some typical benchmarks in this field are tested using GW method, with which the results obtained by other algorithms are compared.
\end{abstract}

\section{Introduction}

Topology optimization for continuum structures is to find the optimal material distribution for structure under given design objectives and constrains in a predetermined design domain. Since the pioneering work of Bendsoe and Kikuchi [1] in 1988, topology optimization for continuous structures has been extensively explored and has already became a frontier of the structural optimization area $[2,3,4]$. Up to now, the practical application scope of topology optimization is continuously extended. Apart from traditional mechanics problems, the method of topology optimization has also been applied to the optimal design of compliant mechanisms[5,6], the design for control of wave propagation $[7,8]$ or acoustic response $[9,10]$ of structures, the design of multifunctional materials $[11,12]$, the optimization of heat transfer $[13,14]$ and fluid flows problems [15,16], etc.. In summary, significant developments of topology optimization at the respects of both modelling methods and solution algorithms have been acquired during the last three decades, and numerous engineering problems have been successfully addressed by these theoretical fruits.

Among those applications we mentioned above, the optimal design of compliant mechanisms shows great promise, especially the design of one important kind of compliant actuators- the MicroElectro-Mechanical Systems (MEMS) [17, 18]. Compliant mechanisms are a kind of mechanisms that gain their mobility via elastic deformation of their constituents, which is totally different with their counterparts- the rigid body mechanisms that gain their mobility from the movement of hinges, sliders and bearings [4]. Compare to rigid body mechanisms, compliant mechanisms have numerous advantages, such as they require fewer components; are easy to manufacture; don't need lubrication during operating; even have no need for motor, and so on.

Due to the compliant mechanism's enormous merits, more and more researchers devoted themselves into studying the design methods of it. Ananthasuresh et al. [5, 19, 20] firstly proposed to extend topology optimization method into the design of compliant mechanisms in 1994, and they took the weighted linear combination of output displacement and strain energy as the objective function. Afterward, various kinds of concrete approaches to the topology optimization of compliant mechanisms emerged. In 1997, Sigmund [6] modelled the output load with a spring, and used mechanical advantage as the objective function; then he solved the formulation with Sequential Linear Programming (SLP). In the same year, Frecker and Ananthasuresh et al. [21] proposed a multicriteria optimization strategy wherein the ratio of output displacement and strain energy was used as the objective function; then they also utilized SLP as the solution algorithm for their mathematical model. 
In consideration of the limitations of MP and heuristic methods and the advantages of OC method, this paper introduces a new OC method- the Guide-Weight method $(\mathrm{GW})$ into the topology optimization of compliant mechanisms. The so-called Guide-Weight method was firstly proposed by Chen [22, 23] for the optimization of large-scale antenna structures in 1980s. Because of its advantages of clear physical meaning, high computational efficiency and strong generality, Li et al.[24] and Liu et al.[25] firstly introduced GW algorithm into topology optimization and it shown good behavior. In 2013, Xu et al.[26] utilized GW to solve the topology optimization problems of continuum structures withstanding body forces, which validated its high efficiency and strong robustness in calculating topology optimization problems.In this paper, we improve the GW method further and employ it into solving the topology optimization of compliant mechanisms, which is able to fully exploit GW's superiority of high convergence speed and strong robustness.

\section{The Guide-Weight algorithm}

Assuming that we establish the topology optimization mathematic model with SIMP, then the typical topology optimization problem for continuum structure can be usually expressed as following

Find : $\quad \rho=\left[\rho_{1}, \rho_{2}, \ldots, \rho_{n}\right]^{T} \in R^{N}$

Min : $\quad f(\rho)$

Subject to: $K \square=F$

$$
\begin{aligned}
& V(\rho)=\sum_{i=1}^{N} \rho_{i} v_{i} \leq \eta V_{0} \\
& 0<\rho_{\min } \leq \rho_{i} \leq 1 \quad i=1,2, \ldots, N
\end{aligned}
$$

To derive the solution formula of eq.(1), the Lagrange function need be constructed at first

$L(\rho)=f(\rho)+\lambda\left(V(\rho)-\eta V_{0}\right)$

where $\lambda$ is the Lagrange multiplier.

According to the GW theory [26], we can get the ultimate iteration formula of the GW algorithm as following

$$
\begin{aligned}
& \rho_{i}^{(k+1)}=\left\{\begin{array}{l}
1, \text { if } \rho_{i} \geq 1 \\
\alpha\left(\frac{G_{i}}{\lambda H_{i}}\right)^{(k)}+(1-\alpha) \rho_{i}^{(k)}, \text { if } \rho_{\min }<\rho_{i}<1 \\
\rho_{\min }, \text { if } \rho_{i} \leq \rho_{\min }
\end{array}\right. \\
& i=1,2, \ldots, N
\end{aligned}
$$

where the superscript $k$ denotes iteration index.

\section{Benchmark examples}

In this section, to illuminate the effect of GW method in solving the topology optimization problems of compliant mechanisms, three typical compliant actuators [4, 6] are topologically optimized by it.

\subsection{Inverter mechanism}

An important kind of compliant actuators is the inverter mechanism, which, as its name suggests, is applied to reverse the direction of input force. It's a basic component for compliant mechanism system, and it could be mounted in series or parallel to accomplish various kinds of force transfer tasks. To clarify the optimizing effect of GW, the design of a simple force inverter is presented. The original design domain and boundary conditions of an inverter mechanism is illustrated at Fig.1, wherein the length $L=80 \mathrm{~mm}$. The objective of this example is to find the optimal topology with maximum output displacement $u_{\text {out }}$ under certain input force $f_{i n}$. The mechanical properties of the structure material distributed in the domain are: Young's modulus $E=1 \mathrm{Mpa}$, Poisson's ratio $v=0.3$. The allowable amount of material is set to be 0.3 , the input load $f_{i n}=1 \mathrm{~N}$, and the penalty factor $p$ is equal to 3. Due to symmetry, only half of the structure is used during practical optimizing procedure, and it is discretized with $N=80 \times 40=3200$ finite elements. 


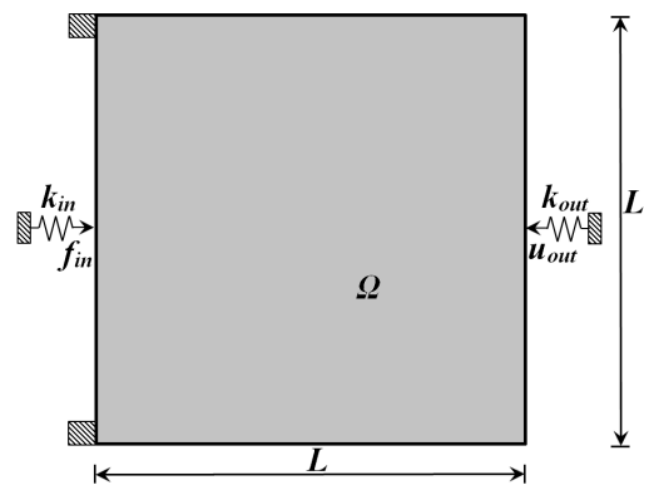

Fig. 1. Design domain and boundary conditions of the inverter mechanism

To study the influence of output port stiffness on optimal topology, $f_{k i n}$ is set to 0.1 and the design problem is solved for various values of $f_{\text {kout }}$. The optimal topologies for $f_{\text {kout }}=0.001,0.01,0.1$, and 1 are depicted in Fig. 2 (a-d), respectively. It's not hard to see that the optimal topology of inverter mechanisms definitely depend on the output spring stiffness, and the structure becomes stiffer with the increase of $f_{\text {kout }}$.

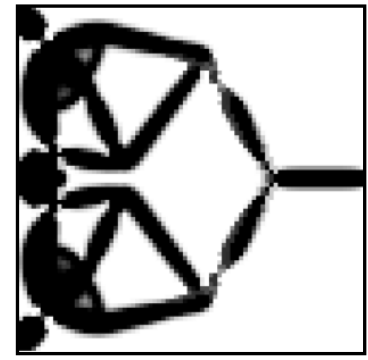

a) $f_{\text {kout }}=0.001$

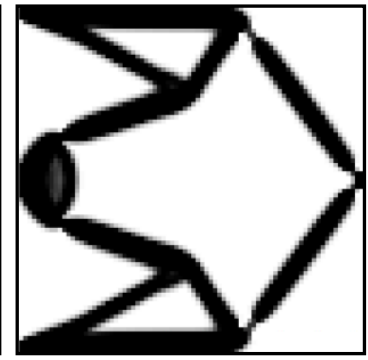

b) $f_{\text {kout }}=0.01$

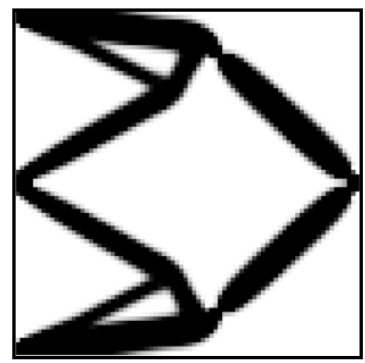

c) $f_{\text {kout }}=0.1$

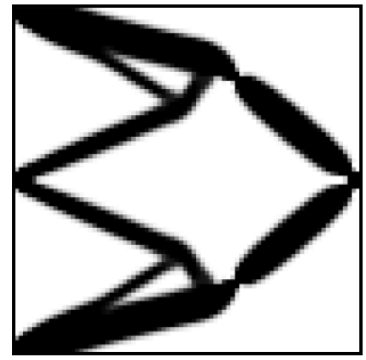

d) $f_{\text {kout }}=1$

Fig. 2. Optimal topologies of inverter mechanisms for fkout $=0.001,0.01,0.1$, and 1 .

Multiplying $K(\text { out, out })_{o}$ of the last iteration by $f_{\text {kout }}$, we can obtain the absolute value of output port spring's stiffness $k_{\text {out }}$. Then corresponding relationship between output displacement, output force and output stiffness are shown in Fig. 3. The theoretical bound is drawn according to that the input work $w_{\text {in }}=f_{\text {in }} \times u_{\text {in }}$ is constant. In order to get a large output displacement, both the mechanism and output port spring must be very soft; on the contrary, stiff structure and spring are needed to yield a large output force. In addition, there is an obvious conflict between output force and output displacement. Therefore, if we want to design a mechanism with high energy efficiency, we need to make compromise between output force and displacement, and specify the value of $k_{\text {out }}$ carefully.

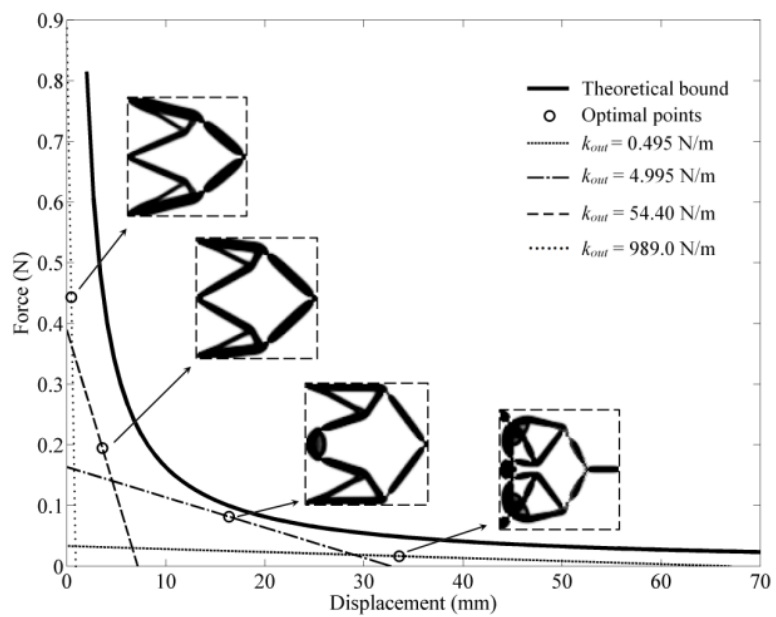

Fig. 3. Force-displacement relationship for the inverter mechanism 


\subsection{Gripper mechanism}

In compliant mechanism field, gripper actuators are used commonly for micromanipulation purpose of gripping. A typical gripper's design domain is shown in Fig. 4. The design domain is a rectangular region, wherein the while and black regions denote void elements and solid elements respectively, whose pseudo density will stay as 0.001 and 1 all the time. There is a jaw at the right side of the structure, which could get its gripping force from the input port at the center of left side. The task of converting a horizontal input force into two vertical forces is namely accomplished by the gripper mechanism. The optimization objective is to maximize the output displacement $u_{\text {out }}$ for constant input force, by distributing material in the grey area. Other necessary parameters are: length $L=80 \mathrm{~mm}$, Young's modulus $E=1 \mathrm{Mpa}$, Poisson's ratio $v=0.3$, the volume constrain fraction is set to be 0.3 , the input load $f_{i n}=1 \mathrm{~N}$, and the penalty factor $p$ is equal to 3 . For the same reason of symmetry, only the lower half of the structure is used to execute optimization, which is discretized with $N=$ $80 \times 40=3200$ finite elements. To facilitate further observation and study, all results will be mirrored back after optimization and analysis.

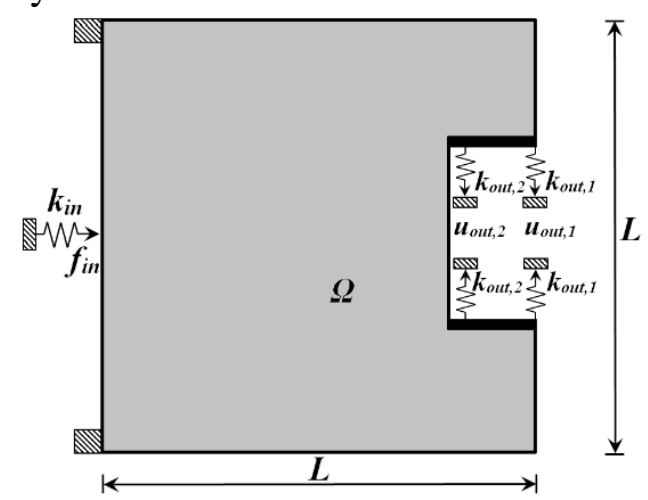

Fig. 4. Design domain and boundary conditions of the gripper mechanism

One simple method of governing the output performance is to just control the outer output port's displacement $u_{\text {out }, 1}$ and its spring stiffness $k_{\text {out }, 1}$, with which method we are able to obtain its topology as showing in Fig. 5a. Corresponding deformation of this kind of optimal design is depicted in Fig.5b, according to which we can see that the two output slabs of the jaw aren't parallel after deformation. This structure couldn't clamp object firmly, thereby it certainly isn't the ideal one we prefer.

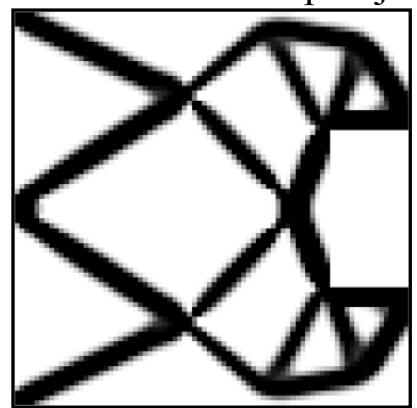

a) Just control uout, 1

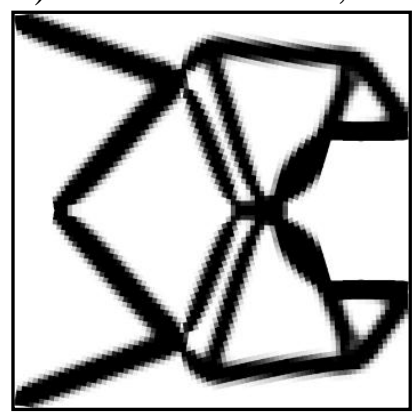

d) Deformation of c)

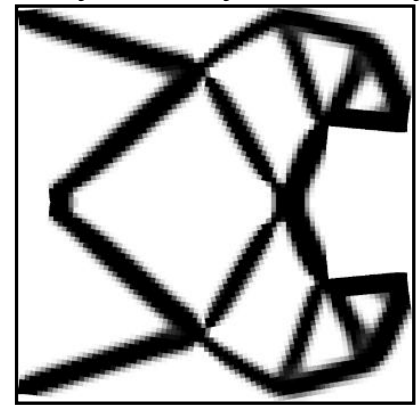

b) Deformation of a)

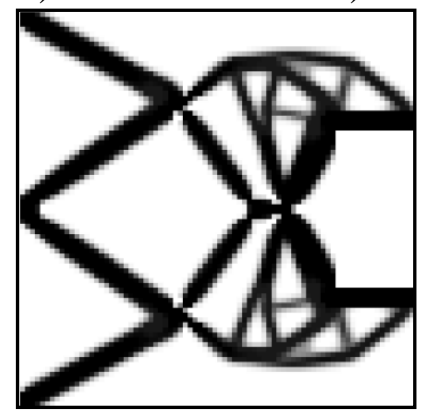

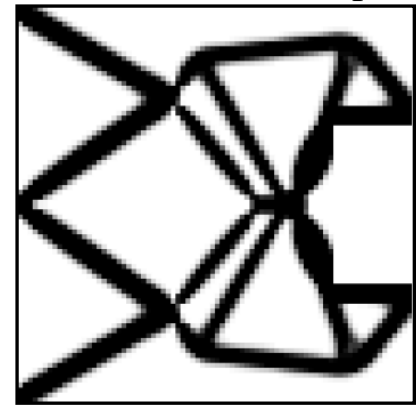

c) Control uout, 1 \& uout,

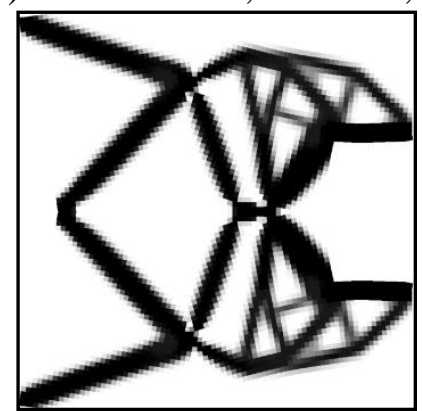

e) Control uout, $1 \&$ uout, 2 in turn f) Deformation of e)

Fig. 5. Optimal topologies and deformation of gripper mechanisms with three different control approaches for output port 


\subsection{Crunching mechanism}

To demonstrate the influence of input force's direction on optimal topology, the design problem sketched in Fig. 6 is considered. The mechanism is subjected to two vertical input forces at the upper and lower right corners. The optimization objective is to maximaze the output force in the horizonal direction of output port. Corresponding parameters are: length $L=90 \mathrm{~mm}$, Young's modulus $E=1$ Mpa, Poisson's ratio $v=0.3$, the volume constrain fraction is set as 0.3 , the penalty factor $p$ is equal to 3 , the amplitude of input load $f_{\text {in }}=1 \mathrm{~N}$. The input and output springs' stiffness ratio $f_{\text {kin }}$ and $f_{\text {kou }}$ are specified as 0.01 and 0.1 respectively. Only the lower half of the structure is used to carry out optimization due to symmetry, which is discretized with $N=90 \times 30=2700$ finite elements.

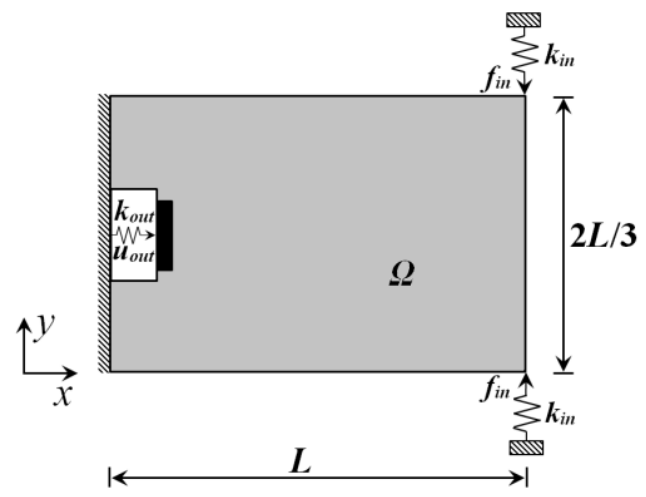

Fig. 6. Design domain and boundary conditions of the crunching mechanism

Firstly, a pressing input force is exerted on the input port, namely, $f_{\text {in }}=-1 N$, then its optimal topology is shown in Fig. 7a. The right half of Fig. 7a structure converts two vertical pressing forces into a horizontal pushing force by its furcate structure. Subsequently, all conditions are kept unchanged, except the directions of two $f_{\text {in }}$ s are reversed, i.e. $f_{\text {in }}=1 \mathrm{~N}$, which means that the optimization object is to maximize the output force while the structure is subjected to two vertical pulling forces. The optimal design in this situation is presented in Fig.7b, in which the right half of structure works like a leverage, whose fulcrum A point reverses two pulling forces into two pressing forces; then the left part of this structure plays the same role as the structure in Fig.7a, namely, converting two pressing forces at $\mathrm{B}$ and $\mathrm{C}$ into a pushing force at $\mathrm{D}$ point. The great distinction between Fig.7b and Fig.7a indicates that the optimal topology of a compliant mechanism heavily depends on the direction of input force, which phenomenon is totally different with the topology optimization of stiff structure.

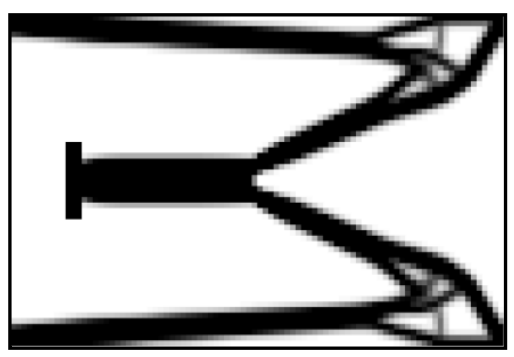

a) $f_{\text {in }}=-1 \mathrm{~N}$

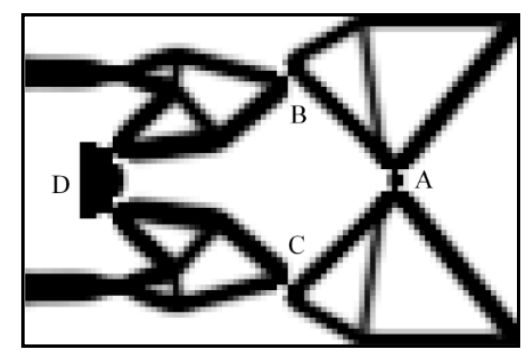

b) $f_{\text {in }}=1 \mathrm{~N}$

Fig. 7. Optimal topologies of crunching mechanisms with pressing and pulling forces

\section{Summary}

In this paper, the theoretical derivation and practical application of the Guide-Weight method for topology optimization of compliant mechanisms are given out. Firstly, the improvement of utilizing self-adaptive modification strategy to set GW's step factor is proved very efficient in solving this type of problems. Secondly, instead of adding absolute value to original element stiffness, relative spring stiffness coefficients are proposed, which is effective in avoiding the local singularity phenomenon of global stiffness matrix, and thereby is able to guarantee iteration convergence more stably and 
robustly. Lastly, three typical benchmarks in this field are successfully optimized, which demonstrate that GW method is not only effective but also efficient in synthesizing compliant mechanisms. Consequently, this paper provides a new idea for researchers to address the topology optimization problems of compliant mechanisms.

\section{Acknowledgements}

The investigation in this paper is supported by National Science Fund for Distinguished Young Scholar (Grant No.51225503), which is gratefully appreciated.

\section{References}

[1] M.P. Bendsoe, N. Kikuchi, Generating optimal topologies in structural design using a homogenization method, Computer Methods in Applied Mechanics and Engineering. 71 (1988) 197-224

[2] H. Eschenauer, N. Olhoff, Topology optimization of continuum structures: A review. ASME Applied Mechanics Review, 54 (2001) 331-390

[3] Deaton J D, Grandhi R V. A survey of structural and multidisciplinary continuum topology optimization: post 2000. Structural and Multidisciplinary Optimization, 2013: 1-38.

[4] M.P. Bendsoe, O. Sigmund, Topology Optimization: Theory, Methods, and Application. Springer, New York, 2003

[5] G.K. Ananthasuresh, S. Kota, Y. Gianchandani, A methodical approach to the design of compliant micromechanisms, Solid-state sensor and actuator workshop, 189-192, 1994

[6] Ole Sigmund, On the design of compliant mechanisms using topology optimization, Mechanics Based Design of Structures and Machines. 25(4), 493-524 (1997)

[7] Sigmund O, Jensen JS, System design of phononic band-gap materials and structures by topology optimization, Philosophical Transactions of the Royal Society A: Physical, Mathematical and Engineering Sciences. 361(1806): 1001-1019 (2003)

[8] Halkjaer S, Sigmund O, Jensen JS, Maximizing band gaps in plate structures, Structural and Multidisciplinary Optimization. 32(4): 263-175 (2006)

[9] Du J, Olhoff N, Minimization of sound radiation from vibrating bi-material structures using topology optimization, Structural and Multidisciplinary Optimization. 34(4-5): 305-321 (2007)

[10] $\mathrm{Du}$ J, Olhoff N, Topological design of vibrating structures with respect to optimum sound pressure characteristics in a surrounding acoustic medium, Struct Multidiscip Optim. 42(1): 43$54(2010)$

[11] Torquato S, Hyun S, Donev A, Optimal design of manufacturable three-dimensional composites with multifunctional characteristics, Journal of Applied Physics. 94(9):5748-5755 (2003)

[12] Chen Y, Zhou S, Li Q, Computational design for multifunctional microstructural composites, International Journal of Modern Physics B. 23: 1345-1351 (2009)

[13] Zhou S, Li Q, Computational design of microstructural composites with tailored thermal conductivity, Numerical Heat Transfer A. 54: 686-708 (2008)

[14] Zhou S, Li Q, Computational design of multi-phase microstructural materials for extremal conductivity, Computational Materials Science. 43: 549-564 (2008)

[15] Borrvall T, Petersson J, Topology optimization of fluids in Stokes flow, International Journal of Numerical Methods in Fluids. 41(1): 77-107 (2003)

[16] Oleson LH, Okkels F, Bruus H, A high-level programming language of topology optimization applied to steady-state Navier-Stokes flow. International Journal of Numerical Methods in Engineering. 7(7): 975-1001 (2006)

[17] O. Sigmund, Design of multiphysics actuators using topology optimization - part I: One material structures, Computer Methods in Applied Mechanics and Engineering. 190: 6577-6604 (2001)

[18] O. Sigmund, Design of multiphysics actuators using topology optimization - part II: Twomaterial structures, Computer Methods in Applied Mechanics and Engineering. 190: 6605-6627 (2001) 
[19] Ananthasuresh, G. K., Kota, S., Kikuchi, N., Strategies for systematic synthesis of compliant MEMS, Dynamic Systems and Control. 2:677-686 (1994)

[20] Ananthasuresh, G. K., A new design paradigm for Micro-Elctro-Mechanical Systems and investigations on the compliant mechanism synthesis, Ph.D. Thesis, University of Michigan, Ann Arbor, MI. (1994)

[21] Frecker M I, Ananthasuresh G K, Nishiwaki S, et al. Topological synthesis of compliant mechanisms using multi-criteria optimization. Transactions of the ASME. 119(2): 238-245 (1997)

[22] Chen Shuxun, Ye Shanghui, Criterion method for the optimal design of antenna structure, Acta Mechanica. Solida. Sinica, 4: 482-497 (1984)

[23] Chen Shuxun, The analysis, combination and optimization design of the system of engineering structures, Chinese Science and Culture Publishing House, Hongkong, China, 2008

[24] Li Zhidong, Liu Xinjun, Guide-Weight method on solving topology optimization problems under single load case, Journal of Mechanical Engineering, 47(15): 107-114 (2011)

[25] X.J. Liu, Z.D. Li, X. Chen, A new solution for topology optimization problems with multiple load: The guide-weight method. Science China Technological Sciences. 54: 1505-1514 (2011)

[26] H.Y. Xu, L.W. Guan, X. Chen et al. Guide-Weight method for topology optimization of continuum structures including body forces, Finite Elements in Analysis and Design. 75: 38-49 (2013) 